\title{
Increased mast cell degranulation and co-localization of mast cells with the NMDA receptor-1 during healing after Achilles tendon rupture
}

\author{
Md. Abdul Alim ${ }^{1,2,3}$ • Paul W. Ackermann ${ }^{2,4}$ - Pernilla Eliasson ${ }^{5}$ - Parmis Blomgran ${ }^{5}$ • \\ Per Kristiansson $^{1} \cdot$ Gunnar Pejler $^{3,6}$ • Magnus Peterson ${ }^{1}$
}

Received: 19 April 2017 / Accepted: 23 August 2017 / Published online: 3 October 2017

(C) The Author(s) 2017. This article is an open access publication

\begin{abstract}
The role of inflammation and the mechanism of tendon healing after rupture has historically been a matter of controversy. The purpose of the present study is to investigate the role of mast cells and their relation to the NMDA receptor-1 (a glutamate receptor) during healing after Achilles tendon rupture. Eight female Sprague Dawley rats had their right Achilles tendon transected. Three weeks after rupture, histological quantification of mast cell numbers and their state of degranulation was assessed by histochemistry. Co-localization of mast cell tryptase (a mast cell marker) and NMDA receptor-1 was determined by immunofluorescence. The intact left Achilles tendon was used as control. An increased number of mast cells and a higher proportion of degranulated mast cells were found in the healing Achilles tendon compared
\end{abstract}

Gunnar Pejler

gunnar.pejler@imbim.uu.se

Magnus Peterson

magnus.peterson@pubcare.uu.se

1 Department of Public Health and Caring Sciences, Family Medicine and Preventive Medicine, Uppsala University, 751

22 Uppsala, Sweden

2 Department of Molecular Medicine and Surgery, Karolinska Institutet, Solna, Sweden

3 Department of Medical Biochemistry and Microbiology, Uppsala University, BMC Box 582, Uppsala, Sweden

4 Department of Orthopedics, Karolinska University Hospital, SE-171 76 Stockholm, Sweden

5 Department of Clinical and Experimental Medicine, Linköping University, Linköping, Sweden

6 Department of Anatomy, Physiology and Biochemistry, Swedish University of Agricultural Sciences, Uppsala, Sweden to the intact. In addition, increased co-localization of mast cell tryptase and NMDA receptor- 1 was seen in the areas of myotendinous junction, mid-tendon proper and bone tendon junction of the healing versus the intact tendon. These findings introduce a possible role for mast cells in the healing phase after Achilles tendon rupture.

Keywords Achilles tendon healing $\cdot$ NMDA $\cdot$ Mast cells · Tryptase $\cdot$ Rats

\section{Introduction}

The physiological mechanisms of tendon healing and pain signaling are still far from understood and the debate continues regarding the role of inflammation in healing and tendinopathy development (Abate et al. 2009; Millar et al. 2017). Patients afflicted by acute Achilles tendon rupture (ATR) also exhibit variable and long-term impaired outcomes such as pain and fatigue (Alim et al. 2016).

In peripheral tissues, the peripheral nerve system (PNS) exhibits a key role in regulating inflammation, pain signaling and healing of the damaged tissue via afferent to efferent pathways (Chiu et al. 2012). Peripheral nerve endings at the site of injury can emit potent neuromediators with the ability to modify the function of fibroblast-like cells in the healing tendon tissue (Ackermann et al. 2014; Murphy and Hart 1993). Emitted neuronal substances may also interact with resident mast cells (MCs) and macrophages to affect their function (Chiu et al. 2012; Murphy and Hart 1993). Conversely, MCs contain substances that, when emitted, may alter the function of both the PNS and tissue cells (Hart et al. 1998). It has been hypothesized that MCs residing near nerve endings may degranulate and affect the 
function of the PNS, which makes them a potential target for modulating inflammation and pain.

Glutamate is the primary excitatory mediator of the nervous system and has, via its preferred receptor, NMDAR1, been implicated in regulating tendon healing (Ackermann et al. 2014; Molloy et al. 2006b) and various pain conditions (Freemont et al. 1997) including tendinopathy (Ackermann et al. 2014). In tendon healing as well as in tendinopathic patients, a 10-fold up-regulation of NMDAR1 expression has been established (Greve et al. 2012; Molloy et al. 2006a). In tendinopathy, NMDAR1 has been observed in morphologically transformed tenocytes in the endothelial and adventitial layers of neovessel walls and in presumed sprouting nerve fibers (Murphy and Hart 1993). The nerve ingrowth in combination with the downstream effect of NMDAR1 activation may represent an important physiological regulation mechanism operative in tendon healing, pain regulation and maybe also in tendinopathy. We hypothesize that there may exist a link between NMDAR1 and MC activation in tendon healing. The aim of this study is therefore to investigate the possible role of MCs in tendon healing. To this end, we used a rat model of ATR and investigated the presence and state of activation of MCs during the healing process. Moreover, the potential communication between MCs and nerve cells was addressed by assessing the spatial relation between MCs and NMDAR1.

\section{Materials and methods}

\section{Study design}

An animal model for ATR was used to quantify and evaluate the localization of the target biomarkers in the healing Achilles tendon (AT) in comparison with the intact AT in the contralateral leg (Eliasson et al. 2009).

\section{Animal model}

Eight female Sprague Dawley rats (Taconic, Ejby, Denmark) were used (13-15 weeks old). The mean weight was $217 \mathrm{~g}$. The animals were housed 2 or 3 per cage with 12:12-h light:dark cycle and given food and water ad libitum. The study was approved by the Linköping Animal Ethics Committee (Ethical number 15-15) for animal experiments and adhered to the institutional guidelines and protocols for the care and treatment of laboratory animals (Eliasson et al. 2009).

\section{Tendon surgery and rupture}

The rats were anesthetized with isoflurane gas (Forene; Abbot Scandinavia, Solna, Sweden) and given antibiotics preoperatively $(25 \mathrm{mg} / \mathrm{kg}$; Oxytetracycline, Engemycin; Intervet, Boxmeer, The Netherlands). Analgesics were given subcutaneously pre- and postoperatively $(0.045 \mathrm{mg} / \mathrm{kg}$; Buprenorphine, Temgesic; Schering-Plough, Brussels, Belgium). The skin on the right lower leg was shaved and cleaned with chlorhexidine ethanol and surgery was performed under aseptic conditions. The tendon complex was exposed through a transverse skin incision lateral to the Achilles tendon. The plantaris tendon was removed and the AT was fully ruptured at the mid-portion of the tendon. The wound was closed with two stitches and the rats were allowed unrestricted activity during healing.

Three weeks after tendon rupture, the rats were euthanized by $\mathrm{CO}_{2}$. The healing tendons were dissected free from extraneous soft tissue and harvested together with parts of the gastrocnemius and soleus muscle complex. The completely dissected tendon and muscle complex was quickly rinsed in saline, snap-frozen in liquid nitrogen and stored at $-80^{\circ} \mathrm{C}$ until tissue slicing. The tendon tissue was sectioned about $12-15 \mu \mathrm{m}$ in size and analyzed by immunohistochemistry and immunofluorescence. The non-ruptured left Achilles tendon was used as control. The plantaris tendon was removed and the Achilles tendon tissue harvested in a similar procedure.

\section{Quantification of mast cell numbers and degranulation}

To assess the numbers of MCs and the degree of degranulation, a semi-quantitative analysis was performed. Tissue samples were stained with toluidine blue solution, $0.1 \%$ toluidine blue (Sridharan and Shankar 2012) in $0.17 \mathrm{mM}$ $\mathrm{NaCl}(\mathrm{pH}=2)$ as previously described (Melo et al. 2015; Sullivan-Brown et al. 2011). Three different regions of interest were marked on the tendon during counting and the total number of MCs and the proportion of degranulated MCs were counted in each field of view using original magnification of $\times 200$. MCs were further divided into cells that were either intact (non-degranulated), partially degranulated, or extensively degranulated (Abd-El-Aleem et al. 2005; Li et al. 2012). All photos were captured using a NikonU brightfield microscope at original magnifications of $\times 100, \times 200$ or $\times 400$.

\section{Histochemistry and immunofluorescence}

For general tissue morphology, a standard histochemistry (hematoxylin and eosin, H\&E staining) protocol was followed to evaluate the basic histological differences between the intact and healing AT (Ahmed et al. 2012; Christensen et al. 2015). The expression and localization of NMDAR1 and MC tryptase was visualized by the immunofluorescence technique. For this, fresh-frozen tissue samples were initially allowed to thaw for $10 \mathrm{~min}$ at room 
temperature. Tissue samples were then washed with phosphate-buffered saline (PBS) for $5 \mathrm{~min}$ and immediately fixed with cold acetone (from $-20^{\circ} \mathrm{C}$ ) followed by immunofluorescence as described (Ackermann et al. 2003). For the NDMAR1 staining, sections were first blocked with normal horse serum, followed by rinsing with PBS $(3 \times)$ and incubatied overnight (room temperature, in the dark) with primary antibody: monoclonal mouse antiNMDAR1 (1:100 in PBS-BSA 0.1\%, Triton 0.3\%; BD Biosciences, Stockholm, Sweden). For tryptase staining, sections were blocked with either normal horse serum (for single tryptase staining) or normal goat serum (for double tryptase/NMDAR1 staining), followed by washing with PBS $(3 \times 5 \mathrm{~min})$. After the first blocking step, sections were blocked to quench non-specific binding of avidin (see final step) using an avidin-biotin blocking kit (Vector Laboratories). After washing, sections were incubated with primary antibody overnight (room temperature, in the dark and in wet conditions). Single staining for tryptase was performed using a mouse monoclonal antitryptase antibody (1:2000 dilution; MAB 1222; $\mathrm{CHEMICON}^{\circledR}$ International). For the double-staining experiments, a rabbit anti-tryptase antiserum was used (1:500 dilution). Next, the tissue sections were washed in PBS ( $3 \times 5 \mathrm{~min}$ ) and then incubated for $60 \mathrm{~min}$ (on a shaker) with biotinylated secondary antibody. As secondary antibody, biotinylated horse anti-mouse Ig (1:250 dilution in PBS-BSA 0.1\%) was used for NMDAR1 staining. For detection of the rabbit anti-tryptase antibody, biotinylated goat-anti rabbit Ig (1:250 dilution in PBS-BSA $0.1 \%)$ was used as secondary antibody. Finally, sections were incubated with streptavidin-Cy2 (for tryptase; 1:2,000; Amersham, Poole, UK)) or streptavidin-Cy3 (for NMDAR1; 1:5,000; Amersham). For the double-staining, tryptase and NMDAR1 staining was performed consecutively to prevent non-specific signals (Christensen et al. 2015). For visualization of nuclei, DAPI (4,6-diamidino2-phenylindole, Invitrogen) staining was performed. Digital images were captured using a Nikon fluorescence microscope (Nikon Eclipse 90i; Japan) equipped with a CCD camera (DS-Qi1 Monochromatic Digital Camera). All photographs were taken at original magnifications of $\times 200$ or $\times 400$.

\section{Statistical analysis}

Data analyses to compare number of mast cells in each region or to compare degranulation state between intact and healing tendons were performed by non-parametric MannWhitney $U$ test using the GraphPad Prism software (v.7.02). A repeated measures 2-way ANOVA with granulation state and region as the two variables was used to analyze if there
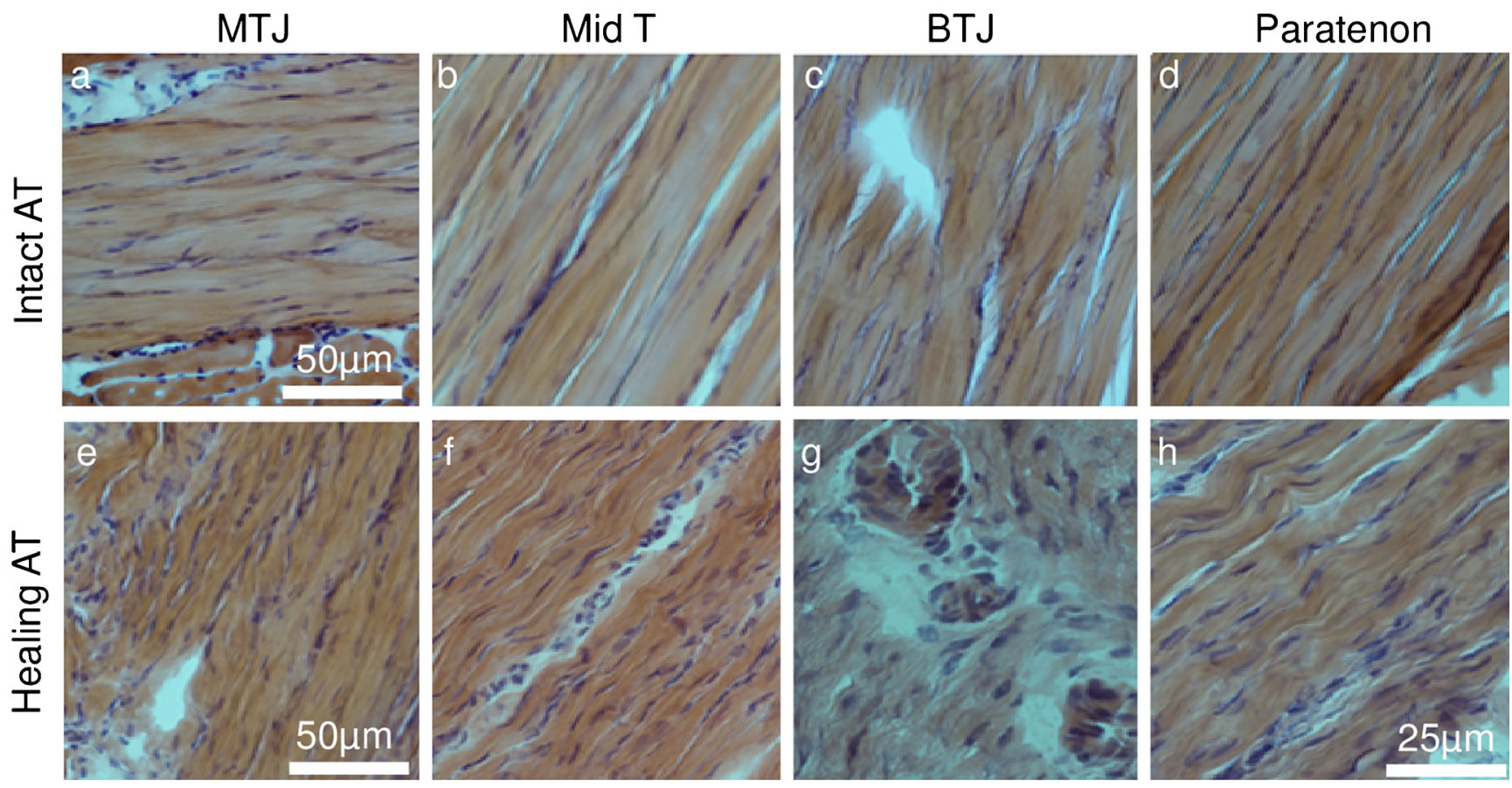

Fig. 1 Basic histological differences in intact and healing Achilles tendon. Haematoxylin and eosin staining of longitudinal sections of intact and healing rat Achilles tendon. The intact Achilles tendon (a-d) exhibits well-organized collagen fibers with a sparse amount of tendon cells, tightly packed between the collagen bundles in four different regions of interest of the tendon: muscle-tendon junction (a), mid-tendon

(b), bone-tendon junction (c) and the paratenon (d). In healing tendon (eh), the structure is disorganized, the collagen becomes wavy, tenocytes change their morphology, the number of cells is increased and vascular ingrowth is seen. Scale bar (b-d, f-h) $25 \mu \mathrm{m}$, (a, e) $50 \mu \mathrm{m}$. MTJ muscletendon junction, Mid T mid-tendon, BTJ bone-tendon junction, $A T$ Achilles tendon 
was a difference in each factor or if there was an interaction between these factors. Intact and healing tendons were analyzed separately. $P$ values $<0.05$ were considered significant.

\section{Results}

\section{Healing tendons exhibit increased cellularity and vascularity}

Intact tendons exhibited normal tissue morphology in the tendon proper, paratenon and other regions of the tendon, i.e., a normal and organized collagen structure was seen, tenocytes were tightly packed between the collagen bundles and no hyper-cellularity or evidence of vascular changes were found in the intact tissue (Fig. 1a-d). Conversely, tissue sections from ruptured AT displayed classical signs of tendon healing, including aberrant cell morphology, increased cellularity, disorganized tissue structure and signs of newly formed blood vessels with vascular ingrowth into the tendon tissue (Fig. 1e-h). These features were apparent in all of the different regions of interest in the tendon after rupture, i.e., muscle-tendon junction (MTJ), mid-tendon, bone-tendon junction (BTJ) and the paratenon.

\section{Increased numbers of degranulated MCs in healing compared to intact AT}

To assess the presence of MCs and their extent of activation, AT tissues were stained with toluidine blue (Fig. 2a). In control, intact tendon, MCs were visible in MTJ, mid-tendon, BTJ and in the paratenon (Fig. 2b; left panels). Moreover, it was observed that a majority of the MCs appeared intact (non-degranulated), i.e., did not show signs of
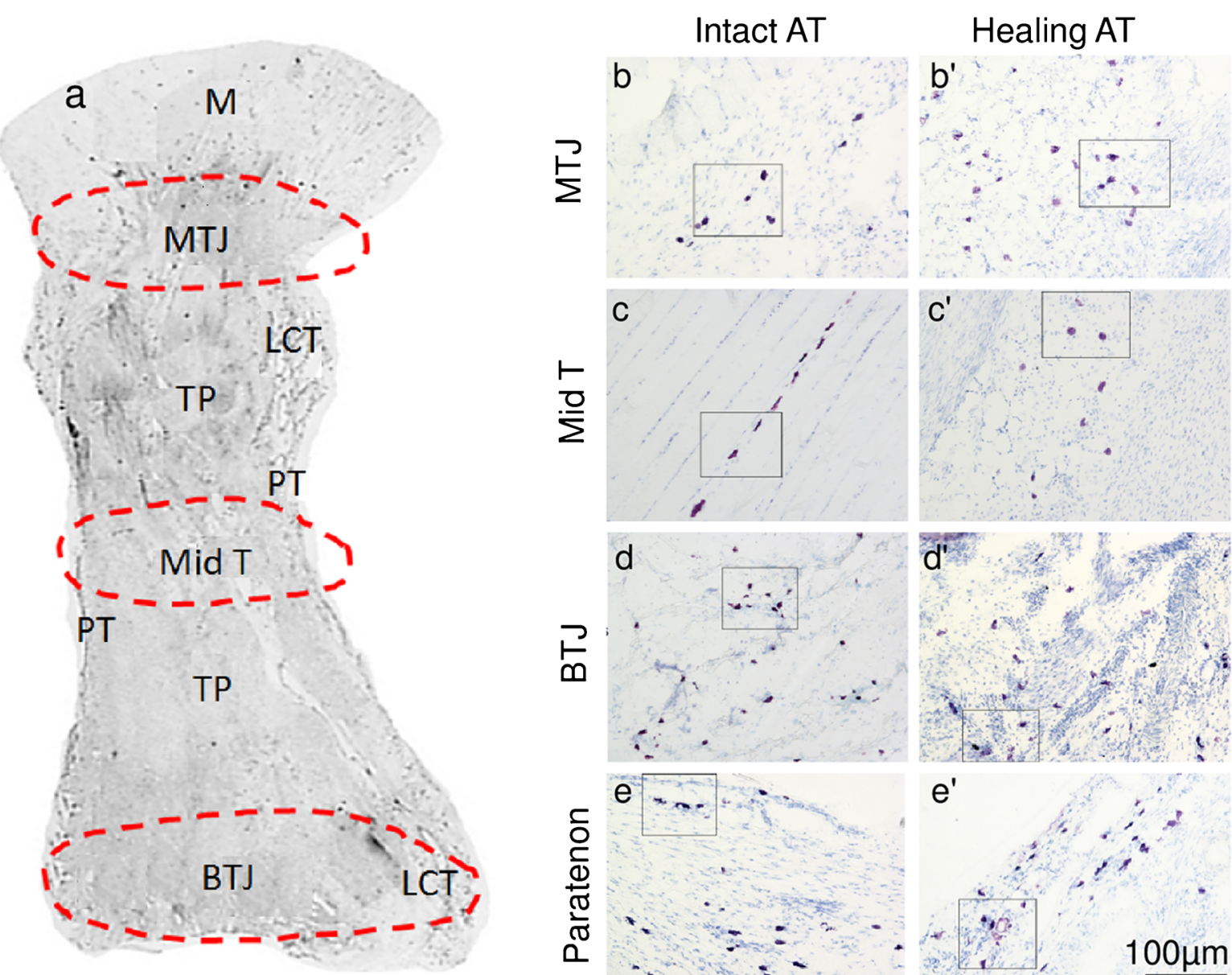

Enlarged
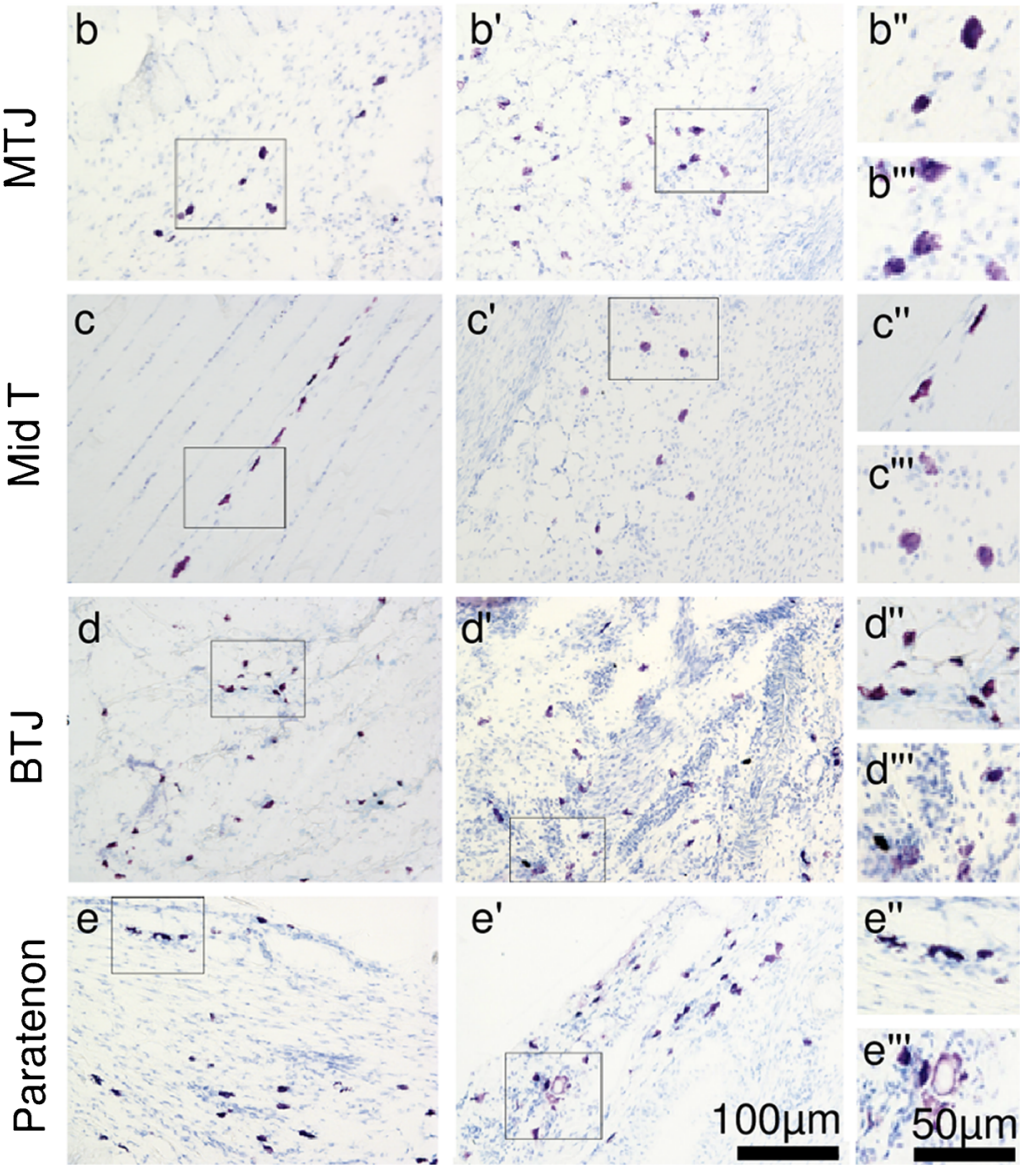

Fig. 2 Mast cell presence and degranulation in intact and healing Achilles tendon. a Anatomical overview of a toluidine blue-stained rat Achilles tendon, with dotted ovals highlighting the areas of interest. The rupture was performed in the mid-tendon region $(0.5 \mathrm{~cm}$ from calcaneal bone). b Toluidine blue staining of the indicated regions of intact or healing Achilles tendon at 3 weeks post-rupture. Magnified portions of

the images are shown to the right. Note the presence of MCs in both the intact and healing tendon. Note also that MCs of healing tendon show signs of activation, as evidenced by degranulation. Scale bars 100 and 50 $\mu \mathrm{m}$. Original magnifications $\times 100$ and $\times 200$. MTJ muscle-tendon junction, Mid T mid-tendon, BTJ bone-tendon junction, $A T$ Achilles tendon, $L C T$ loose connective tissue, $M$ muscle, $P T$ paratenon 
activation (Fig. 2b; left). Notably, the MCs present in the mid-tendon showed a typical linear arrangement (Fig. 2b). In the ruptured tendon, MCs were also found in all different regions of tendon, i.e., MTJ, mid-tendon, BTJ and in the paratenon regions and were frequently found in the vicinity of blood vessels (as highlighted in the lower right panel of Fig. 2b). Whereas MCs of the intact tendon were mainly intact, MCs in the healing tendon were frequently degranulated. This was observed in all of the assessed regions.

Next, the total numbers (intact + degranulated) of MCs in the different regions of the control and ruptured tendon, as well as their state of activation (degranulation) were quantified. As shown in Fig. 3a, the total numbers of MCs were significantly higher in ruptured versus intact MTJ, midtendon and BTJ regions.

To assess the activation state of the MCs, the number of non-degranulated (ND), partially degranulated (PD) and extensively degranulated (ED) MCs were counted (Fig. 3b). These analyses revealed that MCs of both the MTJ, midtendon and BTJ were predominantly in a non-activated (non-degranulated) state in the intact tendon (Fig. 3c, left panel). In contrast, MCs in all these regions of the healing tendon were predominantly degranulated, i.e., showed signs of activation (Fig. 3c, right panel). In total, approximately
$80 \%$ of the MCs in MTJ, mid-tendon and BTJ of the healing tendon displayed degranulation (most of which was extensive), whereas only $20 \%$ of the MCs in the intact tendon were degranulated (Fig. 3d). Collectively, these data indicate that tendon rupture is associated with extensive activation of MCs, in turn suggesting that MCs actively contribute to the processes occurring in response to Achilles tendon rupture.

\section{Mast cell tryptase and NMDAR1 in intact and healing AT}

Previous studies have shown that MCs are often localized in close proximity to nerve endings and it has also been proposed that MC-nerve interaction could be of biological relevance in pathological settings (Gottwald et al. 1998; Li et al. 2012). Based on these notions, we hypothesized that MCs of the healing tendon could potentially communicate with nerve cells, with the possibility that such communication could be of biological significance during the process of tendon healing. To address this possibility, we investigated whether MCs and nerve cells are closely localized within the healing tendon, by immunofluorescence staining for tryptase (MC marker) and NMDAR1 (nerve marker) in adjacent tissue sections. Strong staining for tryptase, using a polyclonal rabbit anti-tryptase antibody, was seen in all assessed areas of the ruptured tendon (MTJ, mid-tendon,
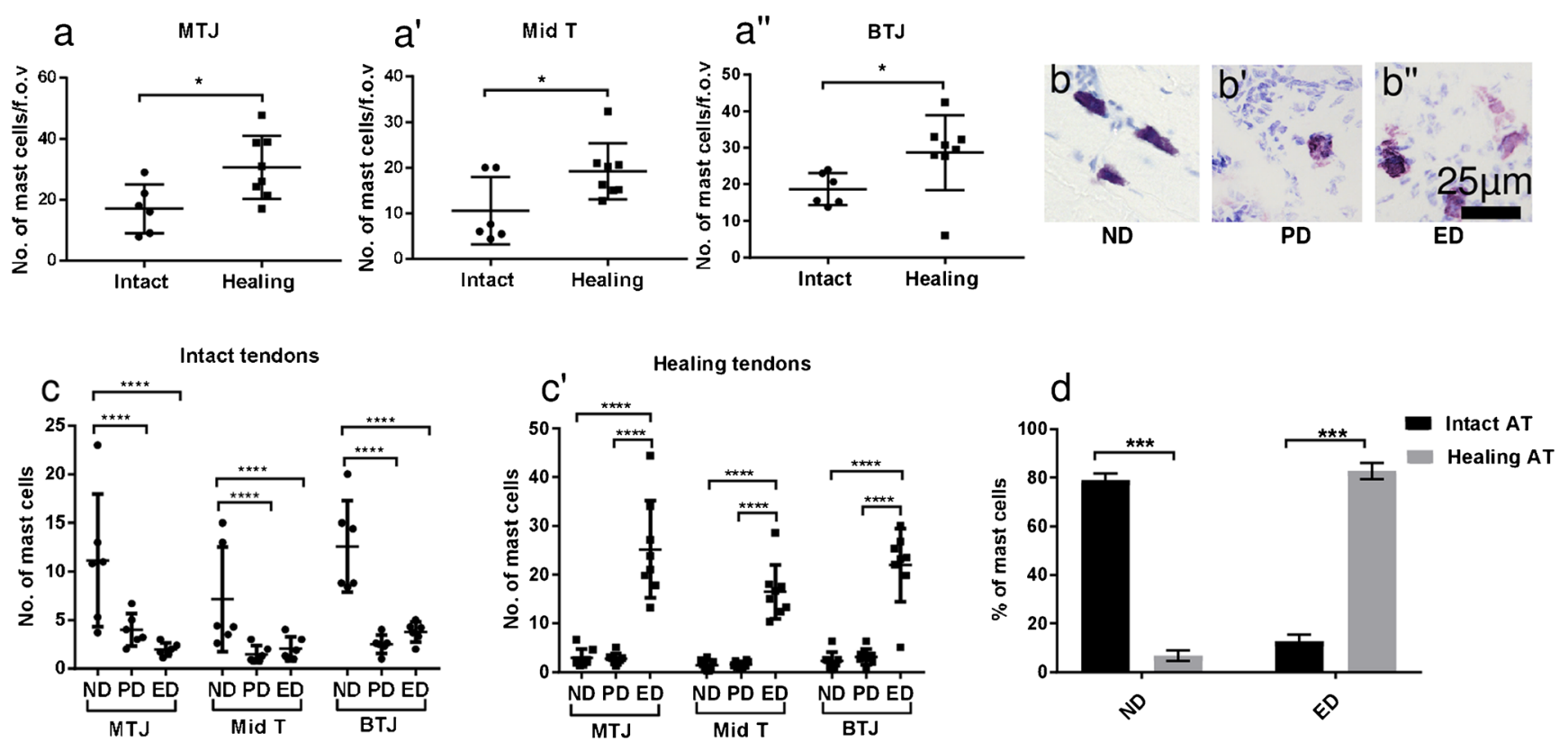

Fig. 3 Number of mast cells and the state of degranulation in intact and healing Achilles tendon. a Total number of mast cells in the muscle-tendon junction, mid-tendon and bone-tendon junction of intact and healing Achilles tendon, based on toluidine blue staining. b Representative images depicting non-degranulated (left panel), partially degranulated (middle panel) and extensively degranulated mast cells (right panel). c Quantification of extensively degranulated, partially degranulated and non-degranulated mast cells in the muscle-tendon junction, mid-tendon and bone-tendon junction of the intact and healing

Achilles tendon. d Percentage of non-degranulated, partially degranulated and extensively degranulated mast cells in the muscle-tendon junction, mid-tendon and bone-tendon junction of intact versus healing tendon. Results are given as mean $\pm \mathrm{SD}$ (intact, $n=6$ and healing, $n=8$ ). $* p<0.05, * * p<0.01, * * * p<0.001, * * * * p<0.0001$. Scale bar $25 \mu \mathrm{m}$. MTJ muscle-tendon junction, Mid T mid-tendon, $B T J$ bone-tendon junction, $A T$ Achilles tendon, $N D$ non-degranulated, $P D$ partially degranulated, $E D$ extensively degranulated, f.o.v. field of view 
BTJ), whereas somewhat weaker staining was seen in the corresponding regions of the intact tendon (Fig. 4a). Immunoreactivity for NMDAR1 was detected in all of the assessed regions of the ruptured tendon but was seen only to a limited extent in the same regions of the intact tendons. Notably, NMDAR1 staining was strong in the mid-tendon of the ruptured tissue, whereas the mid-tendon of intact tissue was essentially negative for NMDAR 1 , indicating that tendon healing after injury is accompanied by upregulation of NMDAR1. To ensure that the staining for tryptase and NMDAR1 was specific, the primary antibodies were replaced with negative control antibodies. Indeed, the staining was completely abrogated when using control primary antibodies, indicating that the staining for tryptase and NMDAR1 was specific (Fig. 4b). Together, these findings indicate that MCs can be found in close proximity to nerve cells in the healing tendon, in support of the hypothesis that MCs and nerve cells may communicate during tendon healing.

\section{Co-localization of MC tryptase and NMDAR1 at the rupture site}

To provide a deeper insight into the possibility that MCs and nerve cells can communicate in the healing tendon, we also performed double immunofluorescence staining for tryptase and NMDAR1. In agreement with the data derived via staining of adjacent sections (see Fig. 4), double immunofluorescence staining revealed that MCs (as marked by tryptase staining) and NMDAR1-positive cells were found closely located in the various regions of the tendon (Fig. 5). In further agreement with the staining of adjacent sections, NMDAR1 positivity was minimal in the mid-tendon of intact tendon whereas strong positivity was seen in the corresponding region of the ruptured tendon. We also noted the presence of cells that were double-positive for tryptase and NMDAR1. Such double positivity was seen in the MTJ, mid-tendon and BTJ of ruptured tendon but was only very scarcely observed in corresponding regions of the intact tendon (Fig. 5). A more detailed analysis
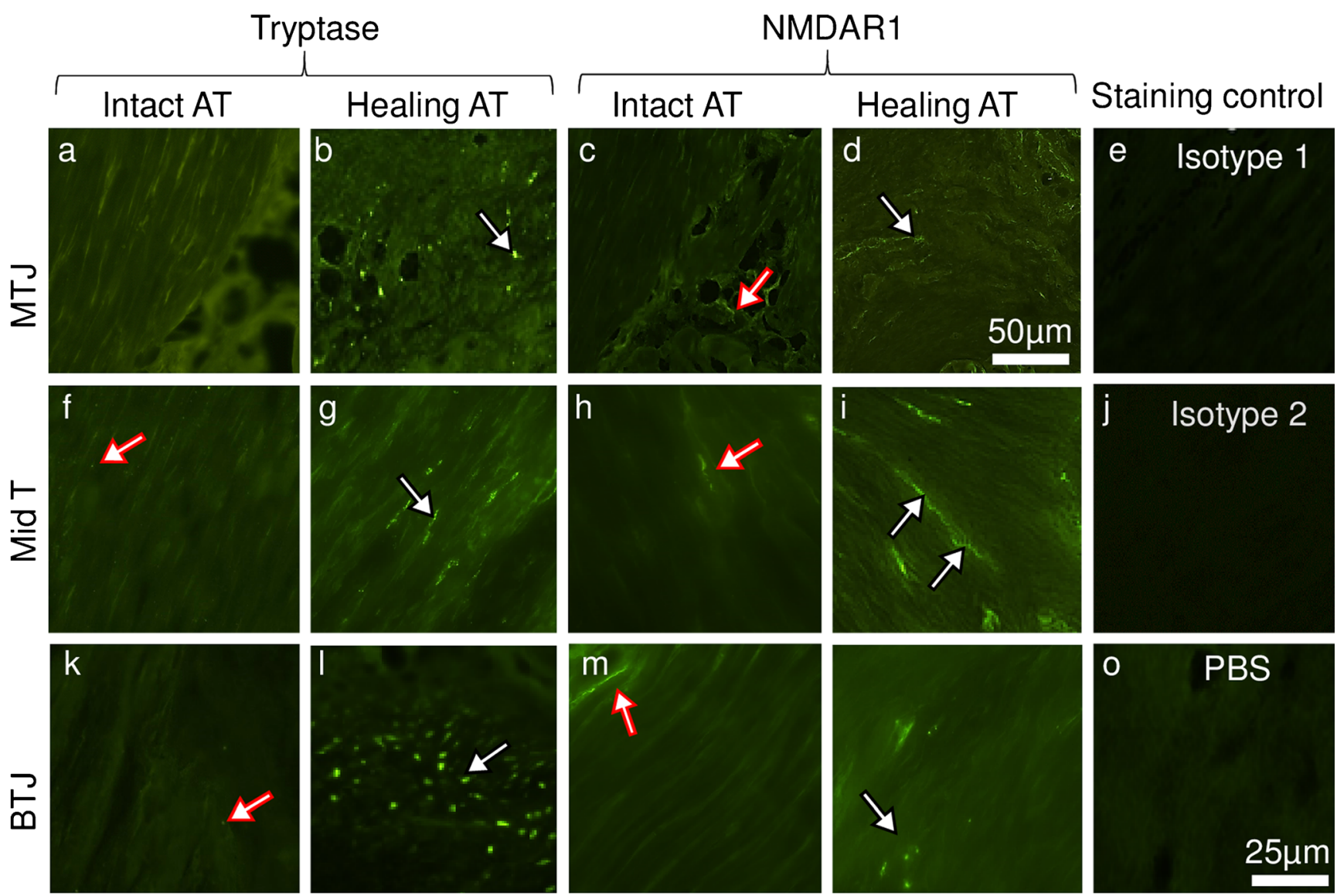

Fig. 4 Immunostaining for mast cells tryptase and NMDAR1 in intact and healing Achilles tendon. a Immunofluorescence micrographs of longitudinal sections from muscle-tendon junction, mid-tendon and bone-tendon junction of intact and healing Achilles tendon. Adjacent sections were stained for tryptase (left panels) and NMDAR1 (right panels). Note the increased tryptase staining in the healing tendon. Note also the increased NMDAR1 positivity of the healing versus intact mid-
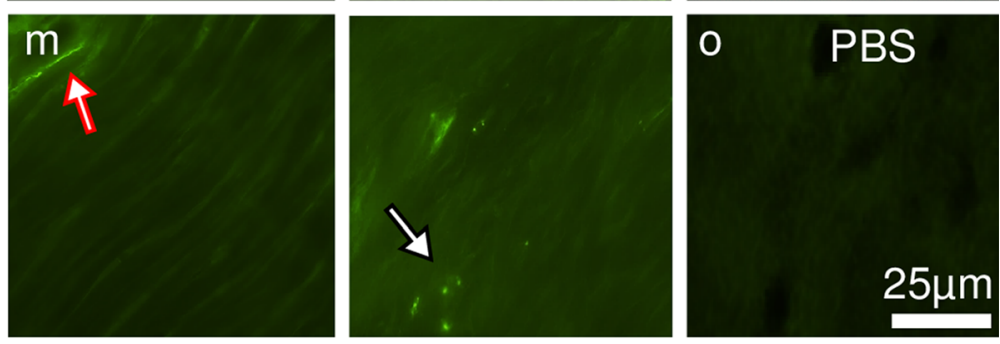

tendon region. b Negative controls for the tryptase (rabbit IgG) and NMDAR1 (mouse IgG1), showing that the tryptase and NMDAR1 staining is specific. Scale bars 25 and $50 \mu \mathrm{m}$. MTJ muscle-tendon junction, Mid $T$ mid-tendon, BTJ bone-tendon junction, $A T$ Achilles tendon, NMDAR1 N-methyl-D-aspartate (NMDA) receptor-1, PBS phosphate buffer saline 

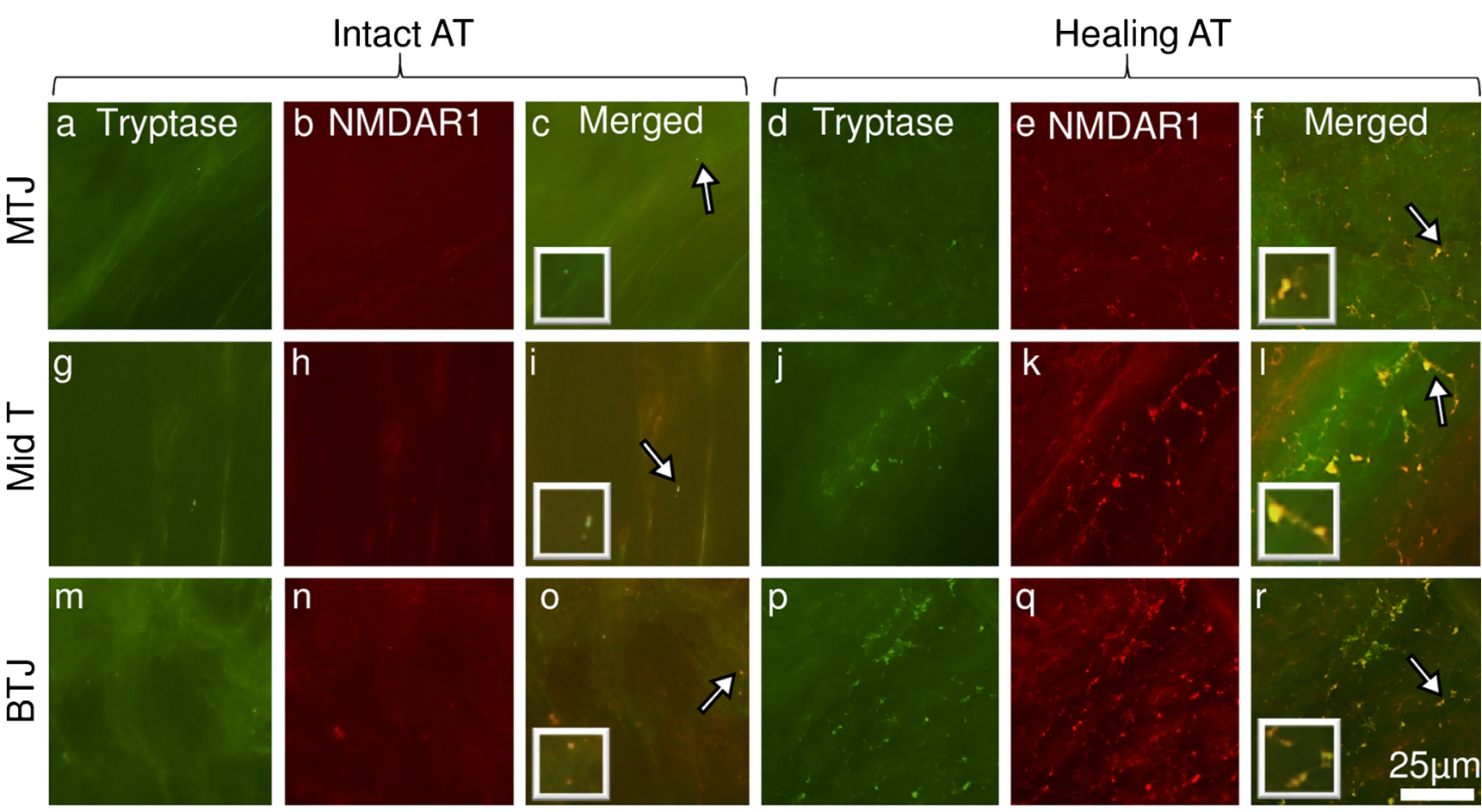

Fig. 5 Co-localization of MC tryptase and NMDAR1. Double immunofluorescence staining for tryptase (green) and NMDAR1 (red) in longitudinal sections from the muscle-tendon junction, mid-tendon and bone-tendon junction of intact and healing Achilles tendon. Note the stronger staining for both tryptase and NMDAR1 in healing vs. intact

Achilles tendon. Note also the co-localization of tryptase and NMDAR1 (magnified in the arrowed area) Scale bars $=25 \mu \mathrm{m}$. MTJ muscle-tendon junction, Mid T mid-tendon, BTJ bone-tendon junction, AT Achilles tendon, NMDAR1 N-methyl-D-aspartate (NMDA) receptor-1

of the callus tissue in the actual area of rupture in the tendon proper was then performed (the ruptured area was identified by tissue morphology). MCs were seen abundantly in the callus area, whereas only limited numbers were seen in the corresponding region of the intact tendon (Fig. 6). Moreover, clear co-localization of tryptase and NMDAR 1 was seen in the ruptured area. Together, these findings indicate that cells of the healing tendon may co-express tryptase and NMDAR1.

\section{Discussion}

MCs have previously been implicated as detrimental players in numerous pathological settings, ranging from allergic disorders including asthma to various other types of pathologies such as cancer, autoimmune disorders and atherosclerosis (Anand et al. 2012; Wernersson and Pejler 2014). In addition, MCs have been suggested to have a number of beneficial functions, e.g., in the context of bacterial and parasite infection, as well as in wound healing (Chiu et al. 2012; Reber et al. 2015). Here, we explored a novel aspect of MCs: their possible involvement in tendon healing in the context of AT rupture.

Our data established that MCs are found in intact AT, contralateral to the ATR in this rat model. However, the numbers of MCs were increased in the healing tendon, after

experimentally-induced rupture. This increase of MCs was particularly prominent in the MTJ but was also seen in the mid-tendon and BTJ. Previous clinical studies based on samples derived from patients suffering from AT rupture have provided limited support for an increase in MC numbers during healing (Scott et al. 2008). Moreover, an increase in MC density has been suggested after rabbit deep flexor tendon repair (Berglund et al. 2010) and in the tendinopathy seen in the calcaneal tendon overuse rat model (Pingel et al. 2013). However, the present study is to our knowledge the first to document increased $\mathrm{MC}$ numbers in experimentally-induced tendon rupture in a rat model for Achilles tendon rupture.

A major finding of this investigation was that MCs of the healing tendon showed signs of activation, as evidenced by extensive degranulation. Notably, MC activation was prominent in all investigated areas of the healing tendon, i.e., MTJ, mid-tendon and BTJ, suggesting that tendon healing is associated with widespread activation of MCs. The consequence of $\mathrm{MC}$ activation in the context of tendon healing and possibly tendinopathy development, is intriguing. When MCs are activated to degranulate, they release a wide panel of preformed mediators from stores in their secretory granules (Wernersson and Pejler 2014). These mediators encompass a number of MC-restricted proteases including tryptase, chymase and CPA3 (Pejler et al. 2010). In addition, MC 

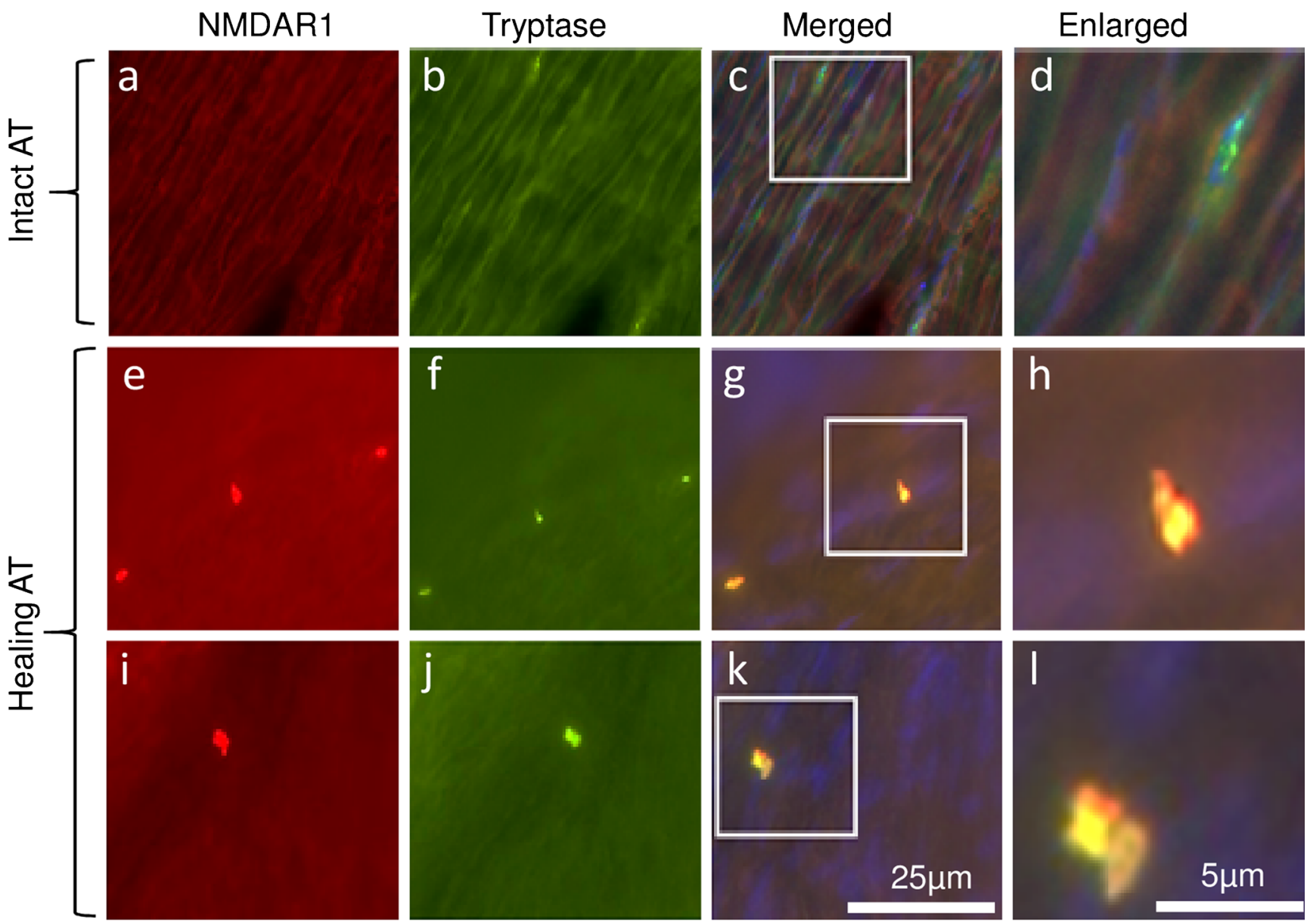

Fig. 6 Co-localization of MC tryptase and NMDAR1 at the site of rupture. Sections were taken from the site of rupture in the mid-Achilles tendon, with the corresponding region of intact Achilles tendon as control. Sections were subjected to double immunofluorescence staining for

tryptase (green) and NMDAR1 (red). Note the co-localization of tryptase and NMDAR1 at the site of rupture. Enlarged images (5 times) are shown to the right. Scale bars 5 and $25 \mu \mathrm{m}$. AT Achilles tendon, NMDAR1 Nmethyl-D-aspartate (NMDA) receptor-1

degranulation causes the release of bioactive amines (e.g., histamine, serotonin, dopamine), proteoglycans, cytokines, growth factors (e.g., TGF-beta, NGF, FGF2, VEGF) and lysosomal hydrolases (Wernersson and Pejler 2014). Potentially, several of the compounds released from activated MCs could influence the inflammatory and proliferative healing phases after tendon rupture. For example, MC-derived growth factors such as TGF-beta (Nakae et al. 2007) and FGF2 could contribute to tissue remodeling processes (Maltby et al. 2009), including stimulation of collagen synthesis (Coussens et al. 1999). Further, MC-derived VEGF (Grutzkau et al. 1998) and NGF may contribute to neo-angiogenesis and nerve ingrowth (Leon et al. 1994) and MC proteases such as tryptase could activate protease-activated receptors on the surface of nerve cells (Akers et al. 2000; Saito and Bunnett 2005).

Another striking finding in this study was that NMDAR 1 and tryptase appear to co-localize in the healing tendon. Tryptase is a MC-restricted protease that is well established as a specific marker for MCs (Castells and Schwartz 1988). Hence, the tryptase-positive cells most likely correspond to
MCs and, consequently, the double tryptase:NMDAR1positive cells may represent MCs that co-express glutamate receptors. To the best of our knowledge, it has not been shown previously that MCs can express such receptors, although there is limited evidence that MCs may respond to glutamate receptor antagonists (Hamasato et al. 2013). However, it is known from previous studies that various other types of immune cells, e.g., neutrophils, can express various glutamate receptors (Gupta et al. 2013) and the present study thus introduces the MC as a novel cell type potentially having the ability to respond to glutamate. The potential consequences of induced NMDAR1 expression in the MCs of the ruptured tendon are not clear at present. However, we may speculate that MCs expressing NMDAR1 can respond to glutamate released from afferent nerve endings, possibly inducing $\mathrm{MC}$ activation and in this way providing an additional amplifying loop to maintain $\mathrm{MC}$ activation and continuous nerve cell engagement. However, further experimental studies are needed to better evaluate the role of a MC-nerve loop. 
Acknowledgements This study was financed by grants from AFA Försäkring (MP) and The Swedish Research Council (GP). We also thank Per Aspenberg for valuable advice.

\section{Compliance with ethical standards}

Conflict of interest The authors declare no conflict of interest in relation to this work.

Open Access This article is distributed under the terms of the Creative Commons Attribution 4.0 International License (http:// creativecommons.org/licenses/by/4.0/), which permits unrestricted use, distribution and reproduction in any medium, provided you give appropriate credit to the original author(s) and the source, provide a link to the Creative Commons license and indicate if changes were made.

\section{References}

Abate M, Silbernagel KG, Siljeholm C, Di Iorio A, De Amicis D, Salini V, Werner S, Paganelli R (2009) Pathogenesis of tendinopathies: inflammation or degeneration? Arthritis Res Ther 11:235

Abd-El-Aleem SA, Morgan C, Ferguson MW, McCollum CN, Ireland GW (2005) Spatial distribution of mast cells in chronic venous leg ulcers. Eur J Histochem 49:265-272

Ackermann PW, Li J, Lundeberg T, Kreicbergs A (2003) Neuronal plasticity in relation to nociception and healing of rat achilles tendon. $\mathrm{J}$ Orthop Res 21:432-441

Ackermann PW, Franklin SL, Dean BJ, Carr AJ, Salo PT, Hart DA (2014) Neuronal pathways in tendon healing and tendinopathy-update. Front Biosci (Landmark Ed) 19:1251-1278

Ahmed AS, Schizas N, Li J, Ahmed M, Ostenson CG, Salo P, Hewitt C, Hart DA, and Ackermann PW (2012) Type 2 diabetes impairs tendon repair after injury in a rat model. J Appl Physiol 113:1784-1791

Akers IA, Parsons M, Hill MR, Hollenberg MD, Sanjar S, Laurent GJ, McAnulty RJ (2000) Mast cell tryptase stimulates human lung fibroblast proliferation via protease-activated receptor-2. Am J Physiol Lung Cell Mol Physiol 278:L193-L201

Alim MA, Svedman S, Edman G, Ackermann PW (2016) Procollagen markers in microdialysate can predict patient outcome after Achilles tendon rupture. BMJ Open Sport Exercise Med 2:e000114

Anand P, Singh B, Jaggi AS, Singh N (2012) Mast cells: an expanding pathophysiological role from allergy to other disorders. Naunyn Schmiedebergs Arch Pharmacol 385:657-670

Berglund ME, Hildebrand KA, Zhang M, Hart DA, Wiig ME (2010) Neuropeptide, mast cell, and myofibroblast expression after rabbit deep flexor tendon repair. J Hand Surg Am 35:1842-1849

Castells M, Schwartz LB (1988) Tryptase Levels in Nasal-Lavage Fluid as an Indicator of the Immediate Allergic Response. J Allergy Clin Immun 82:348-355

Chiu IM, von Hehn CA, Woolf CJ (2012) Neurogenic inflammation and the peripheral nervous system in host defense and immunopathology. Nat Neurosci 15:1063-1067

Christensen J, Alfredson H, Andersson G (2015) Protease-activated receptors in the Achilles tendon-a potential explanation for the excessive pain signalling in tendinopathy. Mol Pain 11:13

Coussens LM, Raymond WW, Bergers G, Laig-Webster M, Behrendtsen O, Werb Z, Caughey GH, Hanahan D (1999) Inflammatory mast cells up-regulate angiogenesis during squamous epithelial carcinogenesis. Genes Dev 13:1382-1397

Eliasson P, Andersson T, Aspenberg P (2009) Rat Achilles tendon healing: mechanical loading and gene expression. J Appl Physiol 107:399-407
Freemont AJ, Peacock TE, Goupille P, Hoyland JA, OBrien J, Jayson MIV (1997) Nerve ingrowth into diseased intervertebral disc in chronic back pain. Lancet 350:178-181

Gottwald T, Coerper S, Schaffer M, Koveker G, Stead RH (1998) The mast cell-nerve axis in wound healing: a hypothesis. Wound Repair Regen 6:8-20

Greve K, Domeij-Arverud E, Labruto F, Edman G, Bring D, Nilsson G, Ackermann PW (2012) Metabolic activity in early tendon repair can be enhanced by intermittent pneumatic compression. Scand J Med Sci Spor 22:E55-E63

Grutzkau A, Kruger-Krasagakes S, Baumeister H, Schwarz C, Kogel H, Welker P, Lippert U, Henz BM, Moller A (1998) Synthesis, storage, and release of vascular endothelial growth factor/vascular permeability factor (VEGF/VPF) by human mast cells: implications for the biological significance of VEGF206. Mol Biol Cell 9:875-884

Gupta R, Palchaudhuri S, Chattopadhyay D (2013) Glutamate induces neutrophil cell migration by activating class I metabotropic glutamate receptors. Amino Acids 44:757-767

Hamasato EK, Ligeiro de Oliveira AP, Lino-dos-Santos-Franco A, Ribeiro A, Ferraz de Paula V, Peron JP, Damazo AS, Tavares-deLima W, Palermo-Neto J (2013) Effects of MK-801 and amphetamine treatments on allergic lung inflammatory response in mice. Int Immunopharmacol 16:436-443

Hart DA, Archambault JM, Kydd A, Reno C, Frank CB, Herzog W (1998) Gender and neurogenic variables in tendon biology and repetitive motion disorders. Clin Orthop Relat Res 351:44-56

Leon A, Buriani A, Dal Toso R, Fabris M, Romanello S, Aloe L, LeviMontalcini R (1994) Mast cells synthesize, store, and release nerve growth factor. Proc Natl Acad Sci U S A 91:3739-3743

Li WW, Guo TZ, Liang DY, Sun Y, Kingery WS, Clark JD (2012) Substance P signaling controls mast cell activation, degranulation, and nociceptive sensitization in a rat fracture model of complex regional pain syndrome. Anesthesiology 116:882-895

Maltby S, Khazaie K, McNagny KM (2009) Mast cells in tumor growth: angiogenesis, tissue remodelling and immune-modulation. Biochim Biophys Acta 1796:19-26

Melo FR, Wernersson S, Pejler G (2015) Induction of mast cell apoptosis by a novel secretory granule-mediated pathway. Methods Mol Biol 1220:325-337

Millar NL, Murrell GA, McInnes IB (2017) Inflammatory mechanisms in tendinopathy - towards translation. Nat Rev Rheumatol 13:110-122

Molloy TJ, Wang Y, Horner A, Skerry TM, Murrell GA (2006) Microarray analysis of healing rat Achilles tendon: evidence for glutamate signaling mechanisms and embryonic gene expression in healing tendon tissue. J Orthop Res 24:842-855

Murphy PG, Hart DA (1993) Plasminogen activators and plasminogen activator inhibitors in connective tissues and connective tissue cells: influence of the neuropeptide substance P on expression. Biochim Biophys Acta 1182:205-214

Nakae S, Ho LH, Yu M, Monteforte R, Iikura M, Suto H, Galli SJ (2007) Mast cell-derived TNF contributes to airway hyperreactivity, inflammation, and $\mathrm{TH} 2$ cytokine production in an asthma model in mice. J Allergy Clin Immunol 120:48-55

Pejler G, Rönnberg E, Waern I, Wernersson S (2010) Mast cell proteases: multifaceted regulators of inflammatory disease. Blood 115:49814990

Pingel J, Wienecke J, Kongsgaard M, Behzad H, Abraham T, Langberg H, Scott A (2013) Increased mast cell numbers in a calcaneal tendon overuse model. Scand J Med Sci Sports 23:e353-e360

Reber LL, Sibilano R, Mukai K, Galli SJ (2015) Potential effector and immunoregulatory functions of mast cells in mucosal immunity. Mucosal Immunol 8:444-463

Saito T, Bunnett NW (2005) Protease-activated receptors: regulation of neuronal function. Neuromolecular Med 7:79-99 
Scott A, Lian O, Bahr R, Hart DA, Duronio V, Khan KM (2008) Increased mast cell numbers in human patellar tendinosis: correlation with symptom duration and vascular hyperplasia. Br J Sports Med 42:753-757

Sridharan G, Shankar AA (2012) Toluidine blue: A review of its chemistry and clinical utility. J Oral Maxillofac Pathol 16:251-255
Sullivan-Brown J, Bisher ME, Burdine RD (2011) Embedding, serial sectioning and staining of zebrafish embryos using JB-4 resin. Nat Protoc 6:46-55

Wernersson S, Pejler G (2014) Mast cell secretory granules: armed for battle. Nat Rev Immunol 14:478-494 